\title{
Evaluación del efecto de Lactobacillus spp. en el desarrollo del intestino delgado en pollos de engorde
}

\section{Lactobacillus spp. effect evaluation in the small intestine development in broiler chickens}

Fecha recepción: 14 de junio de 2015

Fecha aceptación: 19 de octubre de 2015

\author{
Sandra Paola Rodríguez-González ${ }^{1}$
}

Giovanni Moreno-Figueredo ${ }^{2}$

\section{Resumen}

Los probióticos del tipo Lactobacillus spp. son considerados alternativas promisorias para reemplazar los antibióticos como promotores de crecimiento (APC) en las explotaciones avícolas, ya que influyen en el desarrollo poseclosión del tracto gastrointestinal (TGI) y en su ecología microbiana. En este estudio se evaluó la morfometría del intestino delgado en pollos de engorde al suministrar $0.5 \mathrm{~mL} / \mathrm{L}$ de un producto comercial a base de Lactobacillus spp. Se utilizaron 200 pollos machos de un día de edad, de la línea Avian Cobb 48, los cuales se distribuyeron, con un diseño completamente al azar, en dos grupos, denominados control y experimento; ambos grupos fueron mantenidos en condiciones similares de alimentación, manejo y sanidad. A los días 7, 15 y 35 de edad se realizó el sacrificio y la necropsia; se disectaron segmentos de duodeno, yeyuno e íleon para su procesamiento con la técnica de coloración de Hematoxilina-Eosina $(\mathrm{H}-$ E). Los cambios morfométricos de longitud, ancho y cantidad de vellosidades fueron evaluados utilizando un microscopio trinocular con cámara digital Moticam ${ }^{\circledR}$. Los datos se analizaron usando el programa SPSS versión 19; se realizaron comparaciones de los promedios a través de estadística descriptiva y una prueba ANOVA $(p<0,05)$; se observaron diferencias estimadas significativas $(p<0,05)$ a favor del grupo experimento en el desarrollo poseclosión de los segmentos yeyuno e íleon.

Palabras clave: desarrollo intestino; Lactobacillus; pollos de engorde; promotor; vellosidad (DeCS).

\begin{abstract}
The probiotic Lactobacillus spp. type, are considered promising alternatives to replace the use of (GIT) poseclosion development and in their microbial ecology. Thereof in this study the morphometry of the small intestine in broilers to provide with $0.5 \mathrm{~mL} / \mathrm{L}$ of a commercial product based on Lactobacillus spp. were evaluated. 200 male chickens a day old, from the Cobb Avian 48 line were used, distributed

1 M.Sc. Universidad Nacional de Colombia (Medellín, Colombia). sprodriguezg@unal.edu.co.

2 Ph.D. Fundación Universitaria Juan de Castellanos (Tunja, Colombia). gmorenof@jdc.edu.co.
\end{abstract} antibiotics as growth promoters (APC) in poultry farms, due to their influence in the gastrointestinal tract 
under a completely randomized design, in two groups, called control and experiment. Both groups were kept in similar conditions of feeding, management and health care.

At 7, 15 and 35 days old sacrifice and necropsy was performed. Segments from duodenum, jejunum and ileum were dissected for processing with the hematoxylin-eosin $(\mathrm{H}-\mathrm{E})$ coloration technique. The villi morphometric changes in length, width and number were assessed using a trinocular microscope with digital camera Moticam ${ }^{\circledR}$. Data were analyzed using SPSS version 19 program. Comparisons of averages were conducted through descriptive statistics and ANOVA $(p<0.05)$ test. Significant differences $(p<0.05)$ were estimated in favor of the poseclosion development experiment group of the jejunum and ileum segments.

Keywords: broilers chicken; intestine development; Lactobacillus; promoter; villi. 


\section{Introducción}

En los últimos años se han investigado productos que mejoren el desarrollo poseclosión del tracto gastrointestinal de los pollos de engorde, y brinden los mismos beneficios de los antibióticos promotores de crecimiento, pero que no presenten efectos residuales en el producto final. Pelícano et al. (1) reportaron un mayor desarrollo de la altura de la vellosidad en el duodeno, yeyuno e íleon con el uso de probióticos y prebióticos en pollos de engorde a los 21 días de edad.

El uso de probióticos a base de bacterias, como Lactobacillus spp., y Streptococcus bifudus, y de Levaduras, como Saccharomyces boulardii y Saccharomyces cereviceaes, genera una mejor conversión alimenticia y aumento en la ganancia de peso, por acción de las bacterias acidolácticas, que proporcionan nutrientes digeribles y enzimas digestivas, que ayudan al proceso de síntesis y absorción de vitaminas y minerales y al proceso de digestión, lo cual facilita el metabolismo de los alimentos, permitiendo mantener la flora intestinal en equilibrio y evitar la instauración de patógenos que afectan negativamente la salud intestinal del ave (2).

Durante los primeros días de vida, las aves son poco eficientes para digerir proteínas y grasas, debido a la inmadurez de su sistema digestivo; sin embargo, la actividad enzimática intestinal se estabiliza a partir de los 10 a 14 días poseclosión, por lo que todo disturbio estructural y funcional ocasionado en el tejido intestinal del pollo va a interferir en la salud y el desempeño posterior de las aves. Las células epiteliales de la mucosa intestinal se originan en las criptas de Lieberkühn y migran a lo largo de la superficie de las vellosidades hasta la parte superior de estas, siendo expulsadas al lumen en 48-96 horas (3, 4); así, una mayor altura de las vellosidades intestinales se traduce en un aumento no solo de la superficie intestinal, sino también de la actividad de las enzimas del borde en cepillo y de los sistemas de transporte de nutrientes (5), lo que da lugar a una activación de las funciones de digestión y absorción.
A finales de los años cuarenta se observó que las aves alimentadas con productos de fermentación de Streptomyces aureofaciens tenían ganancia de peso corporal mayor, y al buscar en qué producto se encontraban, fueron identificados en múltiples antibióticos, los cuales se usaron como "promotores de crecimiento" que al ser administrados a una dosis subterapéutica por largos períodos producía una ganancia de peso corporal del 5\%; por estas y otras razones se disparó el uso de estos productos, sin tener en cuenta que causan residualidad y resistencia por su uso indiscriminado, convirtiéndose en un problema de salud pública (6). Por lo anterior, la Unión Europea prohibió el uso de antibióticos como promotores de crecimiento en los alimentos para animales, en especial en las explotaciones avícolas, por la resistencia que generaban tanto en medicina humana y veterinaria; a partir de ello se han experimentado nuevas fuentes de aditivos de origen natural que sean inofensivas y que tengan efectos similares a los antibióticos como promotores de crecimiento (APC); por lo tanto, el uso de probióticos, como Lactobacillus spp., puede ser una alternativa, ya que estos productos se basan en propiedades específicas sobre el sistema digestivo, que incluyen la modificación de la flora, reducción de la velocidad de renovación de la mucosa intestinal, desarrollo y maduración poseclosión de criptas y vellosidades intestinales y modulación del sistema inmune gastrointestinal (6).

\section{Materiales y métodos}

El trabajo se realizó en la finca de la Fundación Universitaria Juan de Castellanos, ubicada en el municipio de Soracá, Boyacá, localizado en la zona centro del departamento, a $5^{\circ} 30^{\prime}$ latitud norte y $73^{\circ}$ de longitud oeste de Greenwich y a 2.942 msnm; la temperatura oscila entre los 11 y los 14 ${ }^{\circ} \mathrm{C}$. Los animales del estudio fueron mantenidos siguiendo las condiciones de bienestar animal y cumpliendo con las exigencias del Comité de Bioética de la Facultad de Ciencias Agrarias de la Fundación Universitaria Juan de Castellanos. 
Se utilizaron 200 pollos machos de un día de edad, de la línea Avian Cobb 48, provenientes de una misma incubadora y lote de reproductoras, los cuales fueron distribuidos, bajo un diseño completamente al azar, en dos grupos de estudio, denominados control y experimento; cada grupo, a su vez, se dividió en cinco subunidades de 20 individuos. Al grupo experimento, desde el momento de la recepción, se les suministró en el agua de bebida $0.5 \mathrm{~mL} / \mathrm{L}$ de un producto comercial a base de Lactobacilus spp., durante los primeros quince días de edad.

A los días 7, 15 y 35, de edad antes de suministrar alimento, fueron seleccionadas de forma aleatoria cinco aves de cada grupo, las cuales se sacrificaron por dislocación cráneo-cervical; se procedió a realizar la técnica de necropsia convencional, retirando los órganos del tracto gastrointestinal; se disectaron 2 segmentos de duodeno, yeyuno e íleon (duodeno: desde el píloro hasta la porción distal de la vuelta duodenal; yeyuno: desde la porción distal del giro duodenal al divertículo de Meckel; íleon: desde el divertículo de Meckel hasta el inicio del ciego), para un total de 6 muestras por ave, cada una con una longitud de $2 \mathrm{~cm}$, las cuales se cortaron con tijera recta y fueron mantenidas en formol buferado al $10 \%$ hasta su posterior procesamiento por la técnica de coloración con Hematoxilina Eosina $(\mathrm{H}-\mathrm{E})$, previa inclusión en bloques de parafina (7); el procesamiento de estas láminas se realizó en un laboratorio privado de Patología en la ciudad de Bogotá; una vez obtenidas en su totalidad las láminas histológicas, fueron observadas, medidas y analizadas utilizando un microscopio Model dmwb1-223 Digital Biological Microscop ${ }^{\circledR}$, usando aumento de 4X y 10X, en el Laboratorio de Sanidad Vegetal de la Fundación Universitaria Juan de Castellanos, de la ciudad de Tunja.

Los cambios morfométricos de cantidad se evaluaron contando las vellosidades por campo de microscopio, en aumento de $4 \mathrm{X}$, y estimando un promedio de ellas; la longitud se determinó desde la base de la vellosidad (muscular de la mucosa) hasta su ápice (lumen del órgano) y el ancho de la parte basal; las mediciones se realizaron utilizando la escala de micras $(\mu \mathrm{m})$; los valores se tabularon en promedios.

\section{Resultados}

El grupo al cual se le suministró Lactobacillus spp. presentó 30, 32 y 35 vellosidades a nivel de duodeno, en los días 7, 15 y 35, respectivamente, como se observa en la Figura 1.

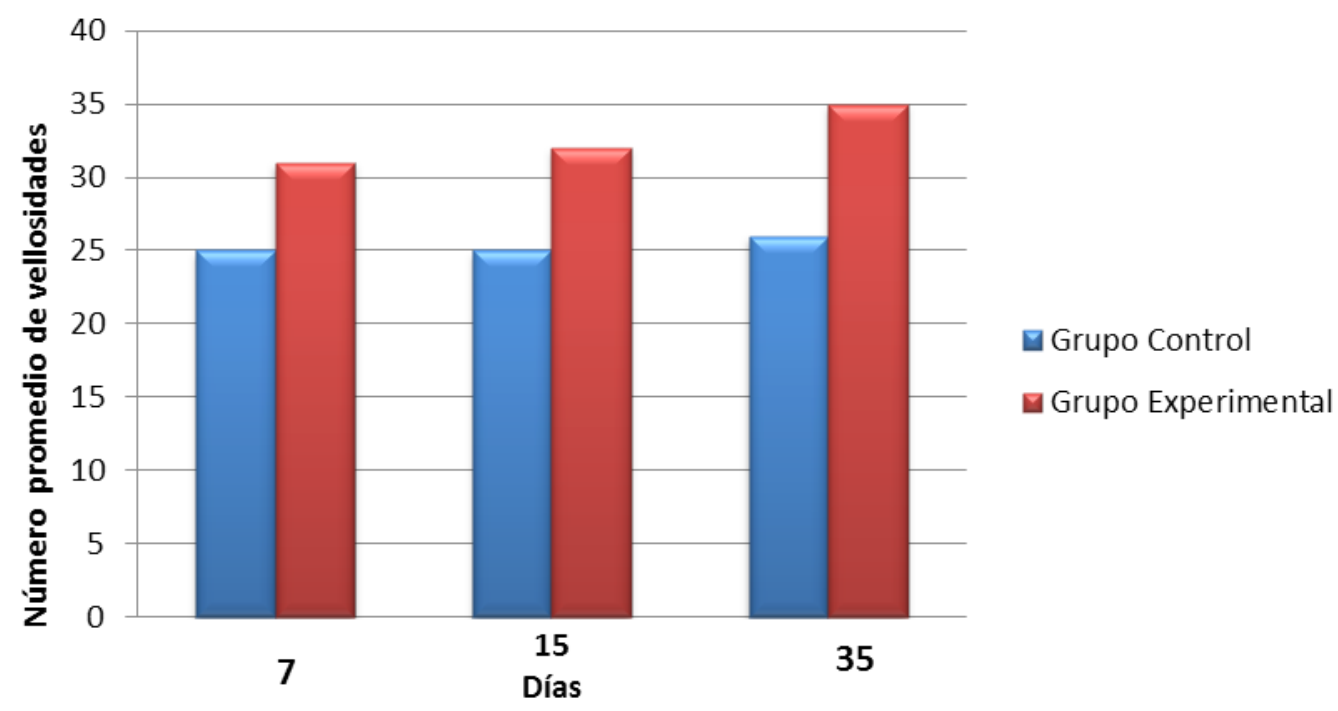

Figura 1. Cantidad de vellosidades a nivel de duodeno para los días 7, 15 y 35 de edad. 
Durante el experimento se observa que el segmento que mayor cantidad de vellosidades presentó, por campo de microscopio, fue a nivel de yeyuno, teniendo un mejor desarrollo a partir del día 15 de edad (Figura 2).

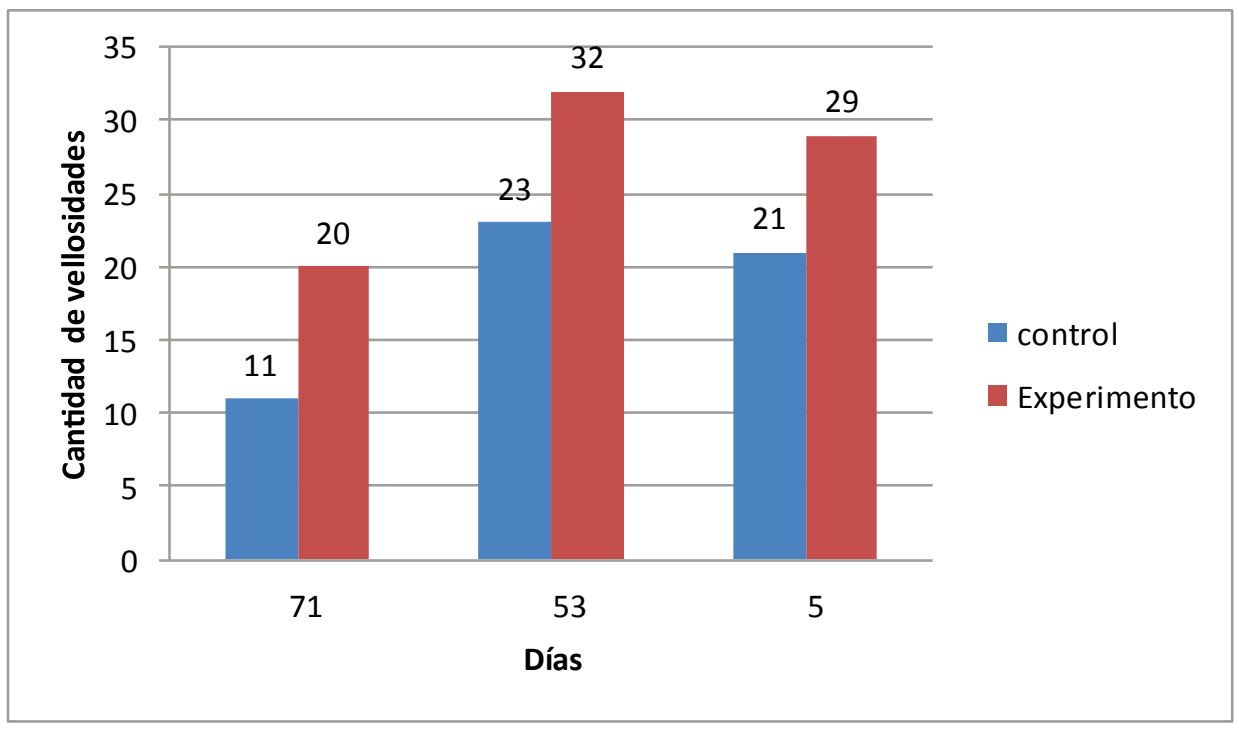

Figura 2. Cantidad de vellosidades a nivel de yeyuno para los días 7, 15 y 35 de edad.

A nivel de íleon se presenta al día 15 de edad un mejor desarrollo de la mucosa, presentando mayor cantidad de vellosidades frente al grupo control, como se observa en la Figura 3.

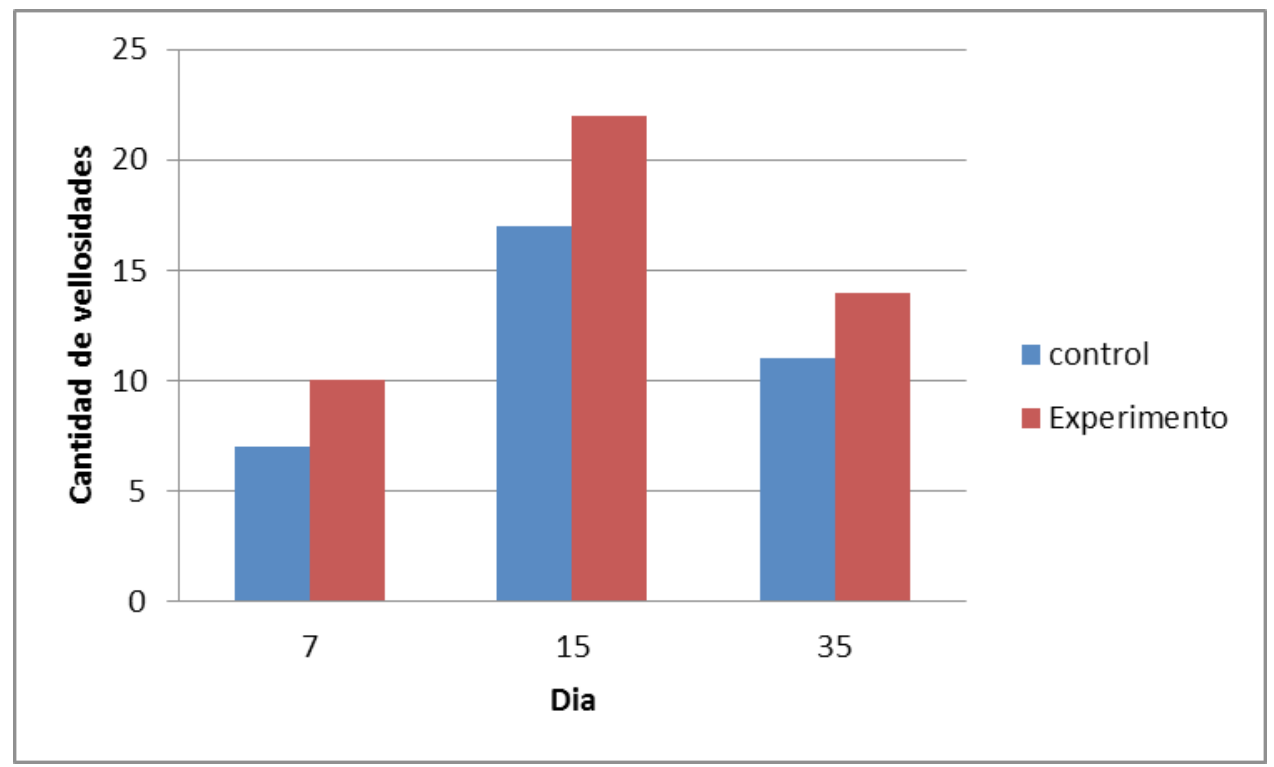

Figura 3. Cantidad de vellosidades a nivel de íleon para los días 7, 15 y 35 de edad.

A nivel duodenal, en el grupo control se presentó un mejor desarrollo longitudinal que en el grupo tratamiento el día $7(1219,5$ vs. $1179,3 \mu \mathrm{m})$ y el día $15(1434,1$ vs. 1340,4 $\mu \mathrm{m})$, pero menor al final del ciclo de producción, como se observa en la Tabla I. 
Tabla I. Medidas morfométricas de la variable longitud en micras para el segmento duodeno.

\begin{tabular}{|c|c|c|c|c|}
\hline & \multicolumn{2}{|c|}{ Medias Morfométricas (Longitud) } & \multicolumn{2}{|c|}{ Expresadas en $\mu \mathrm{m}$} \\
\hline & $\begin{array}{c}\text { Media }(\mu \mathrm{m}) \\
\text { grupo } \\
\text { control }\end{array}$ & $\begin{array}{l}\text { Media }(\mu \mathrm{m}) \\
\text { grupo } \\
\text { tratamiento }\end{array}$ & $\begin{array}{l}\text { Diferencia entre el } \\
\text { grupo control y } \\
\text { tratamiento en } \mu \mathrm{m}\end{array}$ & $\begin{array}{c}\text { \% de la Diferencia } \\
\text { entre el grupo control } \\
\text { y tratamiento }\end{array}$ \\
\hline Día 7 & 1219,5 & 1179,3 & 40,2 & 3,296 \\
\hline Día15 & 1434,1 & 1340,4 & 93,7 & 6,533 \\
\hline Día 35 & 1556,1 & 1755,4 & 199,3 & 12,807 \\
\hline
\end{tabular}

Para la variable de ancho de la vellosidad del duodeno se presentaron diferencias a favor del grupo control durante el tiempo de estudio; datos que se presentan en la Tabla II.

Tabla II. Medidas morfométricas para la variable ancho en micras para el segmento duodeno.

\begin{tabular}{|c|c|c|c|c|}
\hline & \multicolumn{3}{|c|}{ Medias Morfométricas (Ancho) } & Expresadas en $\mu \mathrm{m}$ \\
\hline & $\begin{array}{l}\text { Media }(\mu \mathrm{m}) \\
\text { grupo } \\
\text { control }\end{array}$ & $\begin{array}{l}\text { Media }(\mu \mathrm{m}) \\
\quad \text { grupo } \\
\text { tratamiento }\end{array}$ & $\begin{array}{l}\text { Diferencia entre } \\
\text { el grupo control } \\
\text { y tratamiento } \\
\text { en } \mu \mathrm{m}\end{array}$ & $\begin{array}{c}\text { \% Diferencia entre el } \\
\text { grupo control y } \\
\text { tratamiento en } \mu \mathrm{m}\end{array}$ \\
\hline Día 7 & 159,6 & 149,5 & 10,1 & 6,328 \\
\hline Día15 & 203,9 & 201,6 & 2,3 & 1,128 \\
\hline Día 35 & 265,8 & 250,7 & 15,1 & 5,68 \\
\hline
\end{tabular}

A nivel del segmento yeyuno se presentó mejor desarrollo longitudinal en el grupo experimento al día 7 $(456,24 v s .538,26 \mu \mathrm{m})$ y $15(754,96 v s .666,28 \mu \mathrm{m})$, y para el grupo control el crecimiento longitudinal mejoró al final del ciclo de producción (Tabla III).

Tabla III. Medidas morfométricas de la variable longitud para el segmento del yeyuno en los grupos de estudio.

\begin{tabular}{ccccc}
\hline \multicolumn{4}{c}{ Medias yeyuno (Longitud) } & Expresadas en $\mu \mathrm{m}$ \\
\hline & $\begin{array}{c}\text { Media }(\mu \mathrm{m}) \\
\text { grupo }\end{array}$ & $\begin{array}{c}\text { Media }(\mu \mathrm{m}) \text { grupo } \\
\text { tratamiento }\end{array}$ & $\begin{array}{c}\text { Diferencia entre el } \\
\text { grupo control y } \\
\text { tratamiento en } \mu \mathrm{m}\end{array}$ & $\begin{array}{c}\text { \% de la Diferencia } \\
\text { entre el grupo } \\
\text { control y } \\
\text { tratamiento }\end{array}$ \\
control & 456,24 & 538,26 & 82,02 & 15,23 \\
Día 7 & 764,96 & 666,28 & 98,68 & 14,81 \\
Día 35 & 876,09 & 786,08 & 90,01 & 11,450 \\
\hline
\end{tabular}

Para la variable de ancho de la vellosidad en el segmento yeyuno se presentaron diferencias a favor del grupo experimento en los días 7 y 35 de edad, frente al grupo control (Tabla IV). 
Tabla IV. Medidas morfométricas para la variable ancho en el segmento yeyuno en los grupos de estudio.

\begin{tabular}{lcccc}
\hline \multicolumn{5}{c}{ Medias yeyuno (ancho) Expresadas en $\mu \mathrm{m}$} \\
\hline & $\begin{array}{c}\text { Media }(\mu \mathrm{m}) \\
\text { grupo }\end{array}$ & $\begin{array}{c}\text { Media }(\mu \mathrm{m}) \\
\text { grupo }\end{array}$ & $\begin{array}{c}\text { Diferencia entre el } \\
\text { grupo control y }\end{array}$ & $\begin{array}{c}\text { \% de la Diferencia } \\
\text { entre el grupo } \\
\text { control y } \\
\text { tratamiento }\end{array}$ \\
Día 7 & control & tratamiento & tratamiento en $\mu \mathrm{m}$ & tramient \\
Día15 & 145 & 149,87 & 4,87 & 13,45 \\
Día 35 & 168,19 & 148,25 & 19,94 & 14,38 \\
\hline
\end{tabular}

Se presentó un mejor desarrollo longitudinal para el segmento del íleon en el grupo experimento al día 7 (664,84 vs. $755,41 \mu \mathrm{m}), 15$ (756,83 vs. $834,31 \mu \mathrm{m})$ y 35 (758,23 vs. 905,28 $\mu \mathrm{m})$, (Tablas V).

Tabla V. Medidas morfométricas de la variable longitud para el segmento íleon en los grupos de estudio.

\begin{tabular}{|c|c|c|c|c|}
\hline \multicolumn{5}{|c|}{ Medias íleon (Longitud) } \\
\hline & $\begin{array}{c}\text { Media }(\mu \mathrm{m}) \\
\text { grupo } \\
\text { control }\end{array}$ & $\begin{array}{l}\text { Media }(\mu \mathrm{m}) \\
\text { grupo } \\
\text { tratamiento }\end{array}$ & $\begin{array}{c}\text { Diferencia entre } \\
\text { el grupo control y } \\
\text { tratamiento en } \\
\mu \mathrm{m}\end{array}$ & $\begin{array}{c}\text { \% de la Diferencia } \\
\text { entre el grupo } \\
\text { control y } \\
\text { tratamiento }\end{array}$ \\
\hline Día 7 & 664,84 & 755,41 & 90,57 & 11,98 \\
\hline Día15 & 756,83 & 834,31 & 77,48 & 9,28 \\
\hline Día 35 & 758,23 & 905,28 & 147,05 & 16,24 \\
\hline
\end{tabular}

La Tabla VI muestra los datos de la variable ancho de la vellosidad en el segmento íleon, donde se presentaron diferencias a favor del grupo experimento en los días $15(182,3 v s .191,25 \mu \mathrm{m})$ y $35(201,2$ vs. 205,4 $\mu \mathrm{m}$ ) de edad frente al grupo control, el cual mostró un mejor ancho de la vellosidad en el día 7.

Tabla VI. Medidas morfométricas para la variable ancho para el segmento del íleon en los grupos de estudio

\begin{tabular}{|c|c|c|c|c|}
\hline & \multicolumn{2}{|c|}{ Medias íleon (ancho) } & \multicolumn{2}{|l|}{ Expresadas en $\mu \mathrm{m}$} \\
\hline & $\begin{array}{c}\text { Media }(\mu \mathrm{m}) \\
\text { grupo } \\
\text { control }\end{array}$ & $\begin{array}{l}\text { Media }(\mu \mathrm{m}) \\
\text { grupo } \\
\text { tratamiento }\end{array}$ & $\begin{array}{l}\text { Diferencia entre } \\
\text { el grupo control } \\
\text { y tratamiento } \\
\text { en } \mu \mathrm{m}\end{array}$ & $\begin{array}{c}\text { \% de la } \\
\text { Diferencia entre } \\
\text { el grupo control } \\
\text { y tratamiento }\end{array}$ \\
\hline Día 7 & 166,57 & 163,78 & 2,79 & 1,674 \\
\hline Día15 & 182,3 & 191,25 & 8,95 & 4,679 \\
\hline Día 35 & 201,2 & 205,4 & 4,2 & 2,044 \\
\hline
\end{tabular}

\section{Discusión}

Macari y Luqueti, en 2004, mostraron que el intestino delgado tiene función primordial en los procesos de digestión y, principalmente, en la absorción de nutrientes, ya que gran parte de la función digestiva es debida a la acción de las enzimas (proteínas) pancreáticas: tripsina, quimiotripsina, amilasas, lipasas; en este sentido, en los primeros días de vida del pollo, la actividad pancreática parece ser determinante para digerir substratos en el lumen intestinal, comparado con el desarrollo de los segmentos intestinales, los cuales obtendrían el pico de crecimiento entre los 6 y 8 días de edad. Esto se evidencia en este estudio, donde el segmento yeyuno e íleon presentaron un 
desarrollo entre los días 7 y 15 de edad en las aves del grupo experimento, y mejor desarrollo duodenal en el grupo control, y donde también se observó al microscopio la presencia de vellosidades aun en forma de zigzag en el día 15 de edad para los segmentos duodeno (grupo control) e íleon (grupo experimento), lo que indica que se presentó una mejor absorción de nutrientes en estos segmentos intestinales, ya que las vellosidades dispuestas en esta forma retrasan mejor el paso del alimento por el tracto gastrointestinal que las dispuestas en forma de lengüeta (8). También Velasco et al. Reportaron que las vellosidades organizadas en zig-zag son más eficaces para la absorción de nutrientes que las dispuestas en paralelo o al azar, ya que favorecen un mayor contacto entre el quimo y el epitelio de la mucosa intestinal (10).

Las vellosidades con mejor desarrollo morfométrico en longitud y ancho se presentaron a nivel del yeyuno para el día 15 y 35, y para el íleon, a los días 7, 15 y 35 en las aves suplementadas con Lactobacillus spp.; estos resultados son similares a los hallados por Pelicano et al. (9) y Velasco et al. (10), quienes encontraron vellosidades más altas en este segmento en aves alimentadas con dietas a base de probióticos del tipo Bacillus subtilis, Bacillus licheniformis y Saccharomyces cerevisiae y prebióticos basados en manooligosacáridos (MOS); sin embargo, los resultados no coinciden con los hallazgos obtenidos por (11), quienes no encontraron diferencias para este parámetro entre el grupo control y el grupo al que se le adicionó probióticos a base de Lactobacillus acidophilus y casei, Streptococcus lactis y faecium, Bifidobacterium bifidum y Aspergillus oryzae.

Aunque el estudio de (12), en pollos de 35 días de edad, no encontraron a nivel del yeyuno modificaciones ni en la longitud ni en el ancho, tampoco lo hicieron en la densidad de las microvellosidades por efecto de la incorporación de inulina a la ración. Por el contrario, (13) observaron un incremento en la longitud de las microvellosidades del yeyuno en pollos alimentados con una ración suplementada con frutooligosacaridos (FOS), atribuyéndolo, posiblemente, a la capacidad que tienen estos de crear en el intestino un ambiente microbiano favorable, más que una acción directa sobre el tejido intestinal, lo que se asocia al efecto del Lactobacillus spp. y que se encuentra relacionado con los datos que se evidenciaron en este estudio para el segmento del yeyuno.

En el íleon se observaron vellosidades con disposición en zig-zag aun en el día 15 de edad para las aves con Lactobacillus spp. en el agua de bebida, datos que se asocian al estudio realizado por $(8,14)$, cuando suministraron prebióticos basados en MOS y encontraron vellosidades más largas, aunque los resultados de ese estudio no concuerdan con los hallados aquí, ya que en este no hay diferencias para la cantidad y el ancho de las vellosidades.

Se presentaron diferencias significativas para la variable de ancho a favor del grupo experimento en los tres segmentos intestinales, aunque estudios realizados por (9) no encontraron diferencias entre los grupos que recibieron probióticos y prebióticos en los segmentos intestinales. Estos hallazgos corroboran los resultados parcialmente reportados por (10), en cuanto a que no existe diferencia en el ancho de la vellosidad duodenal y del yeyuno con el uso de probióticos en la ración.

Algunos autores han relacionado estos cambios en las vellosidades con variaciones en la concentración de ácidos grasos de cadena corta (AGCC) en el intestino. Santin et al. (15) señalaron que la formación de estos ácidos grasos a partir de los carbohidratos fermentables es importante para el mantenimiento de la morfología e integridad funcional del epitelio intestinal, ya que en los pollos con suplementación de inulina da lugar a un incremento en las concentraciones de lactato en el yeyuno y butirato en los ciegos (17). Por lo tanto, las aves que consumieron Lactobacillus spp. en el agua de bebida adquirieron un desarrollo morfométrico poseclosión favorable a partir de la segunda semana de edad en los segmentos de yeyuno e íleon.

Williams et al. (16) reportan que el butirato es el de mayor importancia en la relación con la estructura intestinal, ya que es un potente estimulador de la 
división celular; además, se considera que es la principal fuente de energía para los enterocitos y colonocitos, induciendo mayor proliferación celular en las criptas de lieberkühn de la mucosa colorrectal e ileal, lo que se evidenció en las aves del grupo experimento, con un desarrollo morfométrico mejor en este segmento intestinal.

Varios agentes parecen tener acción trófica sobre la mucosa intestinal; dentro de ellos se encuentran: aminas biogénicas, aminoácidos (como glutamina), MOS (mananoligosacáridos) y FOS (frutoligosacáridos), prebióticos, probióticos. Muchos de estos agentes son inductores de mecanismos de transcripción genética por la activación de enzimas importantes en el proceso mitótico en la región cripta-vellosidad; otros tienen acción indirecta, favorecen los mecanismos de proliferación por permitir mayor sanidad en la mucosa intestinal a través de procesos denominados de exclusión competitiva (6), lo que se evidencia en este estudio con el uso de Lactobacillus spp. suministrado desde el momento de la recepción del pollito, ya que este tipo de microorganismos producen un efecto directo sobre la cripta de lieberkünh, favoreciendo el desarrollo de la mitosis de los enterocitos, incrementando la longitud y el ancho de las vellosidades, por el efecto trófico de las proteínas, grasas e hidratos de carbono, ejerciendo funciones sobre la mucosa digestiva como promotores del crecimiento, cubriendo así las necesidades energéticas de los enterocitos, los cuales hacen parte de la homeostasis del lumen intestinal, mejorando la salud y conduciendo a un incremento del número de vellosidades, tal como lo reportan $(9,10)$. El intestino delgado es capaz de aumentar su superficie de absorción no solo mediante su longitud, sino también a través del incremento en el número de sus vellosidades, lo cual se vio reflejado en este estudio para el grupo al cual se le suministró el producto comercial en el agua de bebida.

\section{Conclusiones}

El producto comercial a base de lactobacillus spp., suministrado en el agua de bebida a pollos de engorde, presenta efectos benéficos en cuanto al desarrollo poseclosión del tracto gastrointestinal en ellos, en particular en lo relacionado con los parámetros morfométricos de largo, ancho y cantidad de las vellosidades intestinales.

Las vellosidades en forma de zigzag se presentaron a nivel de duodeno e íleon desde el día 7-15 de edad para el grupo suplementado con lactobacullus spp., lo cual puede favorecer la absorción y aprovechamiento de los nutrientes.

\section{Referencias}

(1) Pelicano E., Souza P., Souza H., Oba A., Norkus E., Kodawara,L., \& Lima, T. 2003. Morfometria e ultra-estrutura da mucosa intestinal de frangos de corte alimentados com dietas contendo diferentes probióticos. Revista Portuguesa de Ciências Veterinárias, 98(547): 125-134.

(2) García A., Ávila D., Rodríguez C. 2009. Evaluación del efecto de microorganismos eficientes en agua de bebida suministrada a pollos Ross x Ross en la granja Tunguavita. Ciencia y Agricultura, 7(1): 83-94. Colombia.

(3) Imondi AR \& Bird FH. 1966. The turnover of intestinal epithelium in the chick. Poultry Science, 45: 142-147. DOI: http://dx.doi. org/10.3382/ps.0450142.

(4) Potten CS. 1998. Stem cells in the gastrointestinal epithelium: Numbers characteristics and death. Philos. Trans. R. Soc. Lond. Biological Sciences. 353: 821-830.

(5) Pluske JR., Tompson MJ., Atwood CS., Bird PH., Williams IH \& Hartmann PE. 1996. Maintenance of villus height and crypt depth, and enhancement of disaccharide digestion and monosaccharide absorption in piglets fed on cows' whole milk after weaning. British Journal Nutrition, 76: 409-422. DOI: http://dx.doi. org/10.1079/BJN19960046.

(6) Macari M., Luquetti B. 2004. Uso de aditivos (Amino Ácidos, Prebióticos y Probióticos) sobre la fisiología gastrointestinal y desempeño 
en pollos. Departamento de Morfología y Fisiología Animal. Universidad Estatal Paulista UNESP. Brasil 1-30.

(7) López N. Afanador G. y Ariza CJ. 2008. Evaluación del efecto de la suplementación de levaduras sobre la morfometría de vellosidades intestinales y productos de la microflora en pollo. Revista de Medicina Veterinaria y Zootecnia, Universidad Nacional de Colombia, 55: 63-76.

(8) Pelicano E., Souza P., Souza H., Leonel F., Zeola N., \& Boiago M. 2004. Productive traits of broiler chickens fed diets containing different growth promoters. Rev. Bras. Cienc. Avic., 6(3): 177-182. DOI: http://dx.doi. org/10.1590/S1516-635X2004000300008.

(9) Pelicano ERL., Souza PA., Souza HBA., Figueiredo DF., Biago MM., Carballo SR. \& Bordon VF. 2005. Intestinal mucosa development in broiler chickens fed natural growth promoters. Brazilian Journal of Poultry Science, 7: 221229. DOI: http://dx.doi.org/10.1590/s1516$635 \times 2005000400005$.

(10) Velasco S., Rodríguez ML., Azueta MC., Rebole A., Ortiz LT. 2010. Los Prebióticos tipo inulina en alimentación aviar. I: Características y efectos a nivel intestinal. Revista Complutense de Ciencias Veterinarias 4(2): 87-104.

(11) Pelicano E., Souza P., Souza H., Leonel F., Zeola N., \& Boiago M. 2007. Morphometry and ultra-structure of the intestinal mucosa of broilers fed different additives. Rev. Bras. Cienc. Avic., 9(3): 173-180. DOI: http://dx.doi. org/10.1590/S1516-635X2007000300006.

(12) Santos EC, Teixeira AS, Freitas RTF, Dias ES, Rodrigues PB, Murgas LDS, Oliveira RFM, Santos EC, \& Gachett NAB. 2004. Uso de pro- motores de crescimento sobre o desempenho e morfometria intestinal de frangos de corte na fase inicial. Anais da 41a Reunião Anual da Sociedade Brasileira de Zootecnia; Campo Grande, MS. Brasil.

(13) Rebolé A., Ortiz LT., Rodríguez M. ${ }^{a}$ L., Alzueta C., Trevi-o J., \& Velasco S. 2010. Effects of inulin and enzyme complex, individually or in combination, on growth performance, intestinal microflora, cecal fermentation characteristics, and jejunal histomorphology in broiler chickens fed a wheat-and barley-bassed diet. Poultry Science, 89: 276-286. DOI: http://dx. doi.org/10.3382/ps.2009-00336.

(14) Xu ZR., Hu CH., Xia MS., Zhan XA, \& Wang MQ. 2003. Effects of dietary fructooligosaccharide on digestive enzyme activities, intestinal microflora and morphology of male broilers. Poultry Science, 82: 1030-1036. DOI: http://dx.doi.org/10.1093/ps/82.6.1030.

(15) Santin E., Maiorka A., Macari M., Grecco M., Sánchez JC., Okada TM, \& Myasaka AM. 2001. Performance and intestinal mucosa development of broiler chickens fed diets containing Saccharomyces cerevisiae cell wall. Journal Applied Poultry Research; 10: 236-244. DOI: http://dx.doi.org/10.1093/japr/10.3.236.

(16) Williams J., Mallet S., Leconte M., Lessire M., \& Gabriel I. 2008. The effects of fructooligosaccharides or whole wheat on the performance and digestive tract of broiler chickens. British Poultry Science, 49: 329-339. DOI: http:// dx.doi.org/10.1080/00071660802123351.

(17) Rehman H., Rosenkranz C., Böhm J., \& Zentek J. 2007. Dietary inulin affects the Faecal fermentation in rats fed oligosachharides (inulin) are modulated by dietary calcium level. American Journal Physiology, 264: 855-862. 